\title{
Generalizing the transition from amplitude to oscillation death in coupled oscillators
}

\author{
Wei Zou, ${ }^{1, *}$ D. V. Senthilkumar, ${ }^{2}$ Aneta Koseska, ${ }^{3}$ and Jürgen Kurths ${ }^{2,4,5}$ \\ ${ }^{1}$ School of Mathematics and Statistics, Huazhong University of Science and Technology, Wuhan 430074, China \\ ${ }^{2}$ Potsdam Institute for Climate Impact Research, Telegraphenberg, Potsdam D-14415, Germany \\ ${ }^{3}$ Department of Systemic Cell Biology, Max Planck Institute of Molecular Physiology, Dortmund D-44227, Germany \\ ${ }^{4}$ Institute of Physics, Humboldt University Berlin, Berlin D-12489, Germany \\ ${ }^{5}$ Institute for Complex Systems and Mathematical Biology, University of Aberdeen, Aberdeen AB24 3FX, United Kingdom
}

(Received 29 September 2013; published 7 November 2013)

\begin{abstract}
Amplitude death (AD) and oscillation death (OD) are two structurally different oscillation quenching types in coupled nonlinear oscillators. The transition from AD to OD has been recently realized due to the interplay between heterogeneity and coupling strength [A. Koseska et al., Phys. Rev. Lett. 111, 024103 (2013)]. We identify here the transition from AD to OD in nonlinear oscillators with couplings of distinct natures. It is demonstrated that the presence of time delay in the coupling cannot induce such a transition in identical oscillators, but it can indeed facilitate its occurrence with a low degree of heterogeneity. Moreover, it is further shown that the AD to OD transition is reliably observed in identical oscillators with dynamic and conjugate couplings. The coexistence of $\mathrm{AD}$ and $\mathrm{OD}$ and rich stable $\mathrm{OD}$ configurations after the transition are revealed, which are of great significance for potential applications in physics, biology, and control studies.
\end{abstract}

DOI: 10.1103/PhysRevE.88.050901

PACS number(s): 05.45.Xt, 87.10.-e

A broad class of collective behavior in real-life systems can be characterized both qualitatively and quantitatively by studying the dynamics of an assembly of coupled nonlinear oscillators [1,2]. Systems of coupled oscillators may often respond nontrivially as a result of the nature of interactions among them and thus produce many interesting and novel self-organized behaviors. One striking emergent phenomenon is oscillation quenching [3,4], which refers to suppression of oscillation under coupling and hence it can serve as an efficient mechanism to control the system's dynamics. Two main manifestations of oscillation quenching, namely, amplitude and oscillation death, can be clearly distinguished based on their different dynamical and emergent nature [4,5].

Amplitude death (AD) denotes the suppression of oscillations manifested through the existence of a single stable homogeneous steady state (HSS). To induce AD, it has been shown that at least one of the following conditions is required: (a) sufficiently disparate frequencies [6], (b) time-delayed coupling [7-9], (c) dynamic coupling [10], or (d) conjugate coupling [11] (for a recent review on $\mathrm{AD}$, see Ref. [3]). In contrast, oscillation death (OD) manifests due to the appearance of a stable inhomogeneous steady state (IHSS) as a result of a symmetry breaking of the system. Two separate branches of the unstable IHSS are stabilized, rendering the oscillators spatially distributed on the upper and the lower levels of the IHSS in the limiting case of two coupled oscillators. The mathematical formulation of OD has been initially given by Prigogine and Lefever for a system of two identical Brusselators coupled via a slow recovery variable [12]. Later, OD was found and analyzed in various chemical and biological systems [13-15] (for a recent review on OD, see Ref. [4]). These two manifestations of oscillation quenching differ from each other significantly: Generally, OD induces heterogeneity in a homogeneous medium due

*zouwei2010@mail.hust.edu.cn to its manifestation as an IHSS, whereas AD results in a homogenous behavior, forcing all of the oscillators to populate the same HSS of the system [4,5]. Apart from their distinct emergent and dynamical nature, $\mathrm{AD}$ and $\mathrm{OD}$ have completely different applications: AD is mainly used as a control mechanism in physical or chemical systems [16,17], whereas the main implications of OD lie in biological systems, since it has been interpreted as a background mechanism of cellular differentiation [18-20] and related to neurological conditions [21,22].

Very recently, it was established that the transition from $\mathrm{AD}$ to OD occurs via a Turing-type bifurcation as a result of the interplay between the heterogeneity of the coupled oscillators and the coupling strength [5] or repulsive interaction [23]. This finding is quite interesting as the diffusive coupling renders the system inhomogeneous rather than uniform, in contrast to the intuitive understandings of its effects [24]. Such diffusion-induced inhomogeneity is perfectly corroborated in the phenomenon of chimera states [25], which surprisingly exist in systems of identical oscillators with homogeneous symmetric coupling.

In this Rapid Communication, we examine the generic nature of the transition between $\mathrm{AD}$ and $\mathrm{OD}$ in nonlinear oscillators with different coupling schemes, namely, timedelayed, dynamic, and conjugate couplings, which have already been shown to induce AD [3]. The generic model of concern in this work is the paradigmatic Stuart-Landau limit-cycle oscillator [1] $\dot{Z}(t)=\left[1+i w-|Z(t)|^{2}\right] Z(t)$. It has an unstable origin and a stable limit-cycle motion $Z=$ $x+i y=e^{i w t}$, where $w$ defines the intrinsic frequency of the limit-cycle oscillation.

Considering two such oscillators coupled via the $x$ component (vector-type coupling) with time delay, the dynamical equations in Cartesian coordinates are expressed as

$$
\begin{aligned}
& \dot{x}_{j}=p_{j} x_{j}-w_{j} y_{j}+K\left[x_{k}(t-\tau)-x_{j}(t)\right], \\
& \dot{y}_{j}=w_{j} x_{j}+p_{j} y_{j},
\end{aligned}
$$


where $j, k=1,2 ; j \neq k ; p_{j}=1-\left|Z_{j}\right|^{2}=1-x_{j}^{2}-y_{j}^{2} ; K$ is the strength of coupling; and $\tau$ is the propagation time delay. Very recently, AD and OD in the coupled system (1) with $\tau=0$ were reported in [5], where a transition from AD to OD occurs due to the interplay between the strength of interaction and the heterogeneity in the frequencies of the coupled oscillators. Generally, the presence of $\tau>0$ in the coupling does not change the steady-state solutions of the coupled system (1), but may switch their stability. Here we are interested in exploring whether such a transition from AD to OD can occur in coupled identical oscillators with time delay.

Obviously, the coupled system (1) has only one HSS solution located at the origin $(0,0,0,0)$, which exists in the whole parameter space. For two identical oscillators $w_{1}=w_{2}=w$, the IHSS solution is given by $P\left(x_{1}^{*}, y_{1}^{*},-x_{1}^{*},-y_{1}^{*}\right)$, with $x_{1}^{*}=-w y_{1}^{*} /\left(w^{2}+2 K y_{1}^{* 2}\right)$ and $y_{1}^{*}= \pm \sqrt{\left(K-w^{2}+\sqrt{K^{2}-w^{2}}\right) / 2 K}$, which arise at $K=$ $\left(w^{2}+1\right) / 2$ via a pitchfork bifurcation. Nevertheless, the IHSS for nonidentical oscillators $w_{1} \neq w_{2}$ for a given frequency mismatch $\Delta=w_{2} / w_{1}$ cannot be derived analytically, however they can be traced numerically. The stable regimes of HSSs and IHSSs of the coupled system (1) are delineated using a MATLAB TRACEDDE package, which employs pseudospectral differentiation techniques and enables one to compute characteristic roots and produce stability charts of linear autonomous delayed systems [26,27]. The onset of AD (a stable HSS) and OD (a stable IHSS) is also confirmed numerically by integrating the coupled system (1) with the fourth-order Runge-Kutta method. Random initial conditions can be adopted when the coupled system (1) experiences $\mathrm{AD}$, as $\mathrm{AD}$ is a unique attractor in the phase space. However, initial conditions near the IHSS are needed for OD because OD is always accompanied by stable limit cycles [4,5].

In the absence of the time delay in the coupling $(\tau=0)$, it has been shown in [5] that, for $w_{1}=2$, the HSS can be stabilized for $K>4.25$ if $\Delta=1$ [see also Fig. 1(a)]. For $1 \leqslant \Delta \leqslant 1.95$, OD appears if $K$ exceeds a certain threshold,

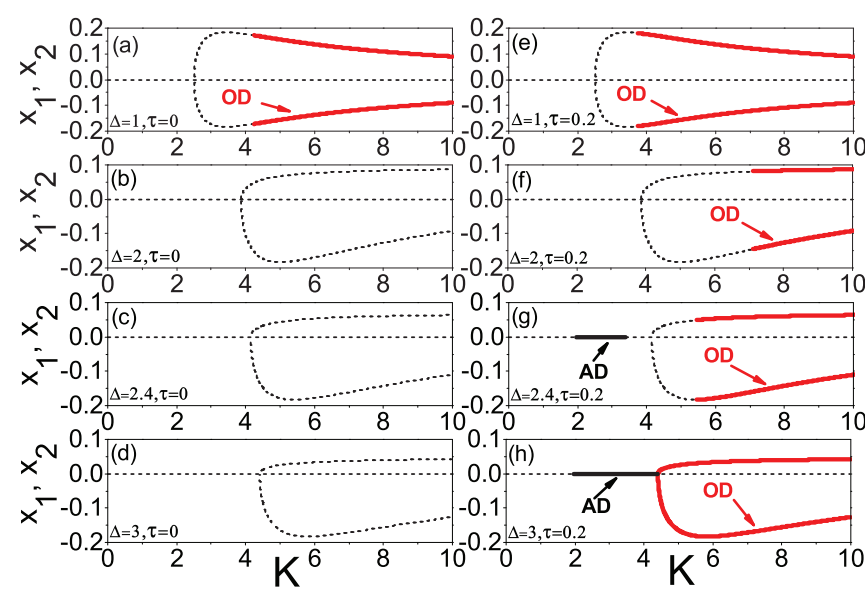

FIG. 1. (Color online) Comparison of the evolution from AD to OD in the coupled system (1). Thin dashed lines denote unstable steady states, whereas solid red (light gray) and black (dark gray) lines denote stable IHSSs (OD) and stable HSSs (AD), respectively. See text for further details. where $\mathrm{AD}$ is impossible for any $K$. In the range of $1.95<$ $\Delta<3.1$, the coupled oscillators no longer suffer either OD or AD. Only $\mathrm{AD}$ is stable in a certain interval of $K$ if $3.1 \leqslant$ $\Delta<3.45$. The transition from AD to OD can be observed if $\Delta \geqslant \Delta_{C}=3.45$ as $K$ is increased. Figure 2(a) shows the systematic characterization of such a transition.

In contrast to this, time-delayed coupling can stabilize both OD and AD, even for the case where no stable IHSS and HSS solutions can be found without time delay. To highlight the effects of time delay, Fig. 1 compares the solution diagrams of steady states for the coupled system (1) with $\tau=0$ (left column) and $\tau=0.2$ (right column) and $\Delta=1,2,2.4$, and 3 (from top to bottom), respectively. $w_{1}=2$ is fixed. Figure 1(e) shows that the IHSS is stabilized for $K>3.75$, which implies that time-delayed coupling can stabilize OD at a smaller coupling strength in coupled identical oscillators $(\Delta=1)$. Even both AD and OD are unstable for the coupled system (1) with $\tau=0$ [Figs. 1(b)-1(d)], the presence of time delay in the coupling can induce stabilization of both regimes [Figs. 1(f)-1(h)]. Increasing the frequency mismatch $\Delta$ results first in the appearance of a stable OD region [Fig. 1(f)] and then both stable $\mathrm{AD}$ and $\mathrm{OD}$ in disconnected intervals of $K$ [Fig. 1(g)]. Finally, a direct transition from AD to OD occurs [Fig. 1(h)] when $\Delta$ is beyond a certain threshold. Thus time-delayed coupling stabilizes not only a HSS (AD) but also an IHSS (OD) and can facilitate the occurrence of the transition from $\mathrm{AD}$ to $\mathrm{OD}$ in coupled oscillators even at a low degree of heterogeneity compared to the instantaneous coupling.

To characterize systematically the AD to OD transition for $\tau>0$, we traced the stability regions of both solutions in the $(K, \Delta)$ plane for distinct time delays as depicted in Figs. 2(a)-2(d). For a small value of time delay, i.e., $\tau=0.1$ [Fig. 2(b)], the distribution of stable AD and OD islets in the $(K, \Delta)$ plane retains a structure that is similar to but larger than that for $\tau=0$ in Fig. 2(a) and the AD to OD transition occurs at a smaller value of $\Delta_{C}=2.78$. For large values of
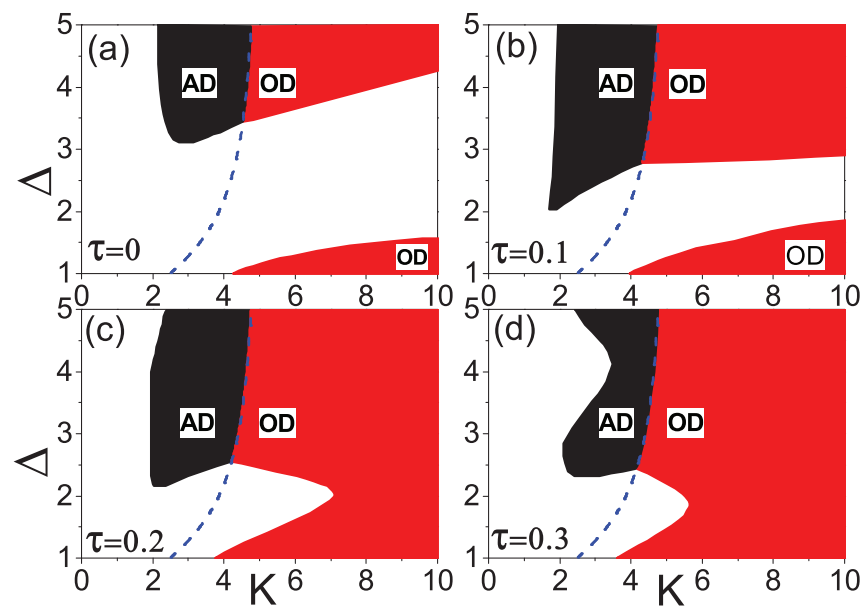

FIG. 2. (Color online) The AD and OD regimes for the coupled system (1) in $(K, \Delta)$ parameter space for (a) $\tau=0$, (b) $\tau=0.1$, (c) $\tau=0.2$, (d) $\tau=0.3$, and $w_{1}=2$. The black (dark gray) and red (light gray) regions denote the stable homogeneous (AD) and inhomogeneous (OD) steady states, respectively. The dashed blue line represents the critical coupling $K_{c}$ for the birth of the IHSS if $K>K_{c}$. 


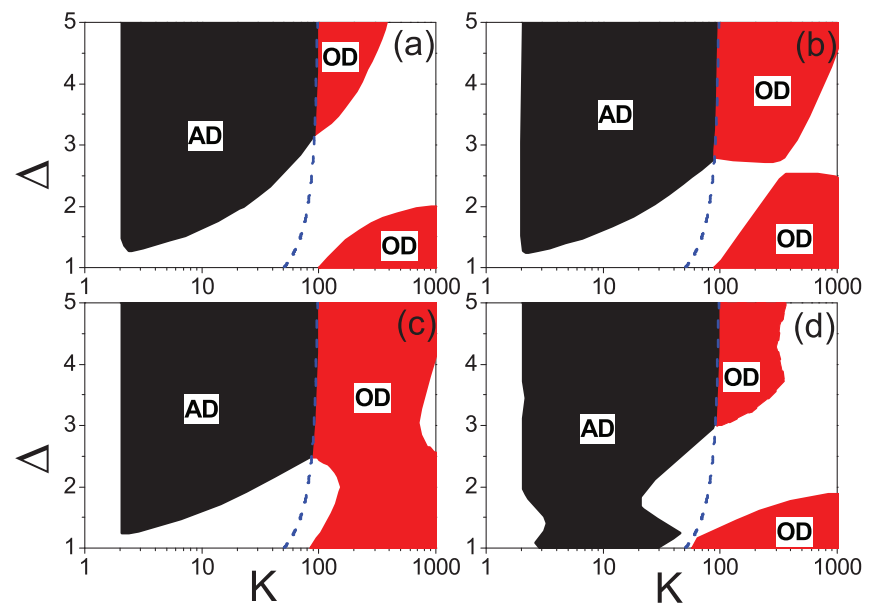

FIG. 3. (Color online) The AD and OD regimes for the coupled system (1) with $w_{1}=10$ in the parameter space of $(K, \Delta)$ for (a) $\tau=0$, (b) $\tau=0.005$, (c) $\tau=0.01$, and (d) $\tau=0.16$.

time delay, the two separate stable OD islets merge into a single connected one and the value of $\Delta_{C}$ for the occurrence of the AD to OD transition is further decreased to $\Delta_{C}=2.56$ for $\tau=0.2$ [Fig. 2(c)] and $\Delta_{C}=2.52$ for $\tau=0.3$ [Fig. 2(d)].

The above analysis reveals that the interplay between the heterogeneity $(\Delta>1)$ and the coupling strength $(K>0)$ is a necessary ingredient to facilitate the AD to OD transition in systems of coupled oscillators, even if the coupling is time delayed. However, the degree of heterogeneity necessary to induce such a transition can be strongly reduced when $\tau>0$. Now it is natural to ask the following question: Can time delay facilitate the AD to OD transition in a system of coupled identical $\left(\Delta_{C}=1\right)$ oscillators as well? If possible, it would mean that a transition between a HSS and a symmetry-breaking IHSS can be realized in a homogeneous medium, which may be of significant importance for control studies and also have potential applications in biological systems.

It is not difficult to numerically find that the minimum intrinsic frequency $w_{\min } \approx 6.25$ is the threshold for the occurrence of delay-induced $\mathrm{AD}$ in the system of coupled identical units (1). Thus $w>w_{\min }$ is a necessary condition to induce a possible AD to OD transition in coupled identical oscillators with time delay. To understand whether it is possible to realize this scenario, now, we investigate the implications of time-delayed coupling when $w_{j}>w_{\text {min }}$.

Figures 3(a)-3(d) depict the stable regimes of AD and OD of the coupled system $(1)$ with $w_{1}=10$ in the $(K, \Delta)$ parameter space for different $\tau$. By taking into consideration a time delay in the coupling, effects on the stable $\mathrm{AD}$ and $\mathrm{OD}$ regimes similar to those in the case of lower intrinsic frequencies (Fig. 2) can be observed [Figs. 3(a)-3(c)]. However, a characteristic time delay $\tau=0.16$ in Fig. 3(d) facilitates the stable AD islet to extend to the $\Delta=1$ axis for a finite range of $K$, where initially only a stable OD region existed for larger coupling strengths. Note that the stable AD and OD islets do not merge with each other and thus the direct transition from AD to OD does not occur at $\Delta=1$.

To obtain the complete picture of the role of time delay in the transition scenario between both dynamical regimes, we further plot the $\mathrm{AD}$ and $\mathrm{OD}$ stability regions in the
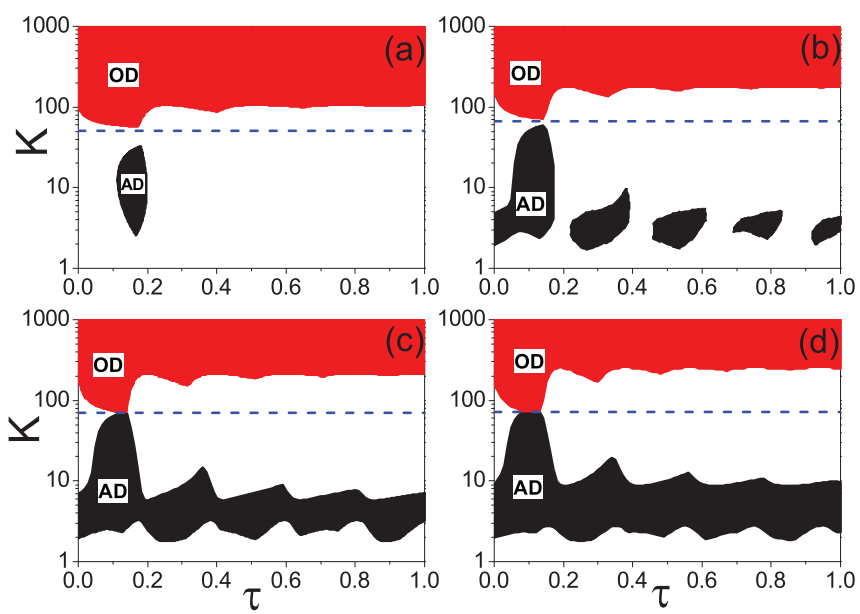

FIG. 4. (Color online) The AD and OD regions for the coupled system (1) in the parameter space of ( $\tau, K$ ) for (a) $\Delta=1$, (b) $\Delta=1.4$, (c) $\Delta=1.5$, and (d) $\Delta=1.6$ with $w_{1}=10$.

$(\tau, K)$ parameter space for various $\Delta$ in Figs. 4(a)-4(d) for $w_{1}=10$. The $\mathrm{AD}$ and $\mathrm{OD}$ islets are clearly separated for $\Delta=1$ [Fig. 4(a)]. The stability regions merge with each other, allowing for a direct AD to OD transition only if $\Delta>$ $\Delta_{C}=1.5$ [comparing Figs. 4(b)-4(d)]. Note that there is a pronounced interval of time delay for which the stable AD and OD regions are connected when $\Delta>1.5$; e.g., see Fig. 4(d) with $\Delta=1.6$. Hence we can conclude that the AD to OD transition occurs due to the interplay of both the heterogeneity and the coupling strength of the system even in the presence of time delay in the coupling. Thus time-delayed coupling cannot induce such a transition in identical oscillators, but it can facilitate its appearance for small values of $\Delta$.

We ask the next question whether other coupling schemes exist that would allow for an $\mathrm{AD}$ to $\mathrm{OD}$ transition in a system of coupled identical oscillators. Recent investigations have revealed that $\mathrm{AD}$ can be induced in identical oscillators with dynamic [10] and conjugate couplings [11], even in the absence of time delay. However, OD has not been observed in both coupling types so far, which may serve as candidates to induce the $\mathrm{AD}$ to $\mathrm{OD}$ transition in identical oscillators.

Coupling two Stuart-Landau oscillators with dynamic coupling [10] results in

$$
\begin{aligned}
& \dot{x}_{j}=p_{j} x_{j}-w y_{j}+K\left(u_{j}-x_{j}\right) \\
& \dot{y}_{j}=w x_{j}+p_{j} y_{j}, \quad \dot{u}_{j}=-u_{j}+x_{k} .
\end{aligned}
$$

Note that, interestingly, the coupled system (2) has the same steady states as those of the two delay-coupled identical oscillators in (1). Here, however, the HSS (origin) is stable for a certain interval of $K$ if $w>2 \sqrt{2}$ [10]. Further, without loss of generality, we adopt $w=5$ and AD occurs for $\frac{1}{2}\left(w^{2}-w \sqrt{w^{2}-8}\right)<K<\frac{1}{2}\left(1+w^{2}\right)$. At $K=\frac{1}{2}\left(1+w^{2}\right)$, a pitchfork bifurcation gives rise to two additional stable branches of the IHSS [Fig. 5(a)], i.e., OD appears. Thus we can state that an AD to OD transition can be indeed realized in identical oscillators with dynamic coupling. 


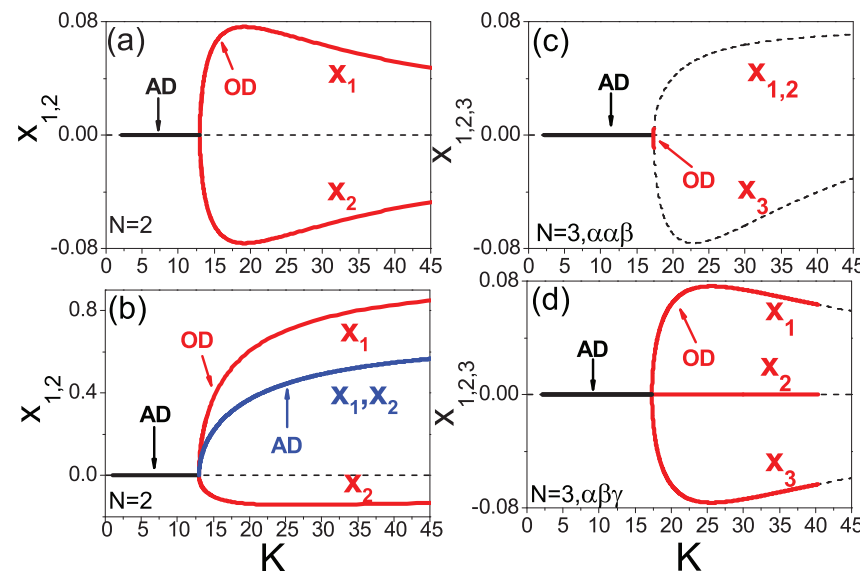

FIG. 5. (Color online) Transition from AD to OD in two coupled identical Stuart-Landau oscillators for $w=5$ with (a) and (b) dynamic and conjugate couplings, respectively, and (c) and (d) three Stuart-Landau oscillators with dynamic coupling for the $\alpha \alpha \beta$ and $\alpha \beta \gamma$ OD patterns after the transition. [11]

For two Stuart-Landau oscillators with conjugate coupling

$$
\begin{aligned}
& \dot{x}_{j}=p_{j} x_{j}-w y_{j}+K\left(y_{k}-x_{j}\right), \\
& \dot{y}_{j}=w x_{j}+p_{j} y_{j}+K\left(x_{k}-y_{j}\right),
\end{aligned}
$$

apart from the HSS at the origin $x_{1}^{*}=y_{1}^{*}=x_{2}^{*}=y_{2}^{*}=0$, other steady states are given by the condition $x_{1}^{*}+y_{1}^{*}=x_{2}^{*}+y_{2}^{*}=$ $K\left(y_{1}^{*} y_{2}^{*}-x_{1}^{*} x_{2}^{*}\right) / w$, from which both HSS and IHSS solutions may be newly created by the coupling. In particular, the HSS at the origin is stabilized for $1<K<\frac{1}{2}\left(1+w^{2}\right)$ if $w>1$. Figure 5(b) illustrates steady states for $w=5$ as a function of $K$. In this case, the origin is destabilized at $K=\left(1+w^{2}\right) / 2$ and besides the two separate stable branches of the IHSS solution (OD), we have also found the existence of an additional stable HSS. Thus the conjugate coupling can induce not only an AD to OD transition in a system of coupled identical oscillators, but after the transition, a new stable HSS (AD) is found to coexist with the stable IHSS (OD).

Furthermore, the transition from AD to OD can be observed for the general case of $N(N>2)$ identical oscillators. We illustrate this with $N=3$ identical Stuart-Landau oscillators with dynamical coupling. The evolution equations are similar to the coupled system (2) with the coupling term replaced by $\dot{u}_{j}=-u_{j}+\left(x_{k}+x_{l}\right) / 2$, where $j, k, l=1,2,3$ and $i \neq j \neq l$. Now, for the case of the IHSS, two different configurations $\alpha \alpha \beta$ and $\alpha \beta \gamma$ are possible. In the first case, two oscillators populate the same branch, while the third oscillator occupies the opposite branch of the IHSS ( $\alpha \alpha \beta$ distribution), whereas in the second case, all oscillators populate different branches of the IHSS ( $\alpha \beta \gamma$ distribution). We note here that the observation of such a three-branch manifestation of OD is lacking in the literature to the best of our knowledge. It should be noted that the patterns form any circular permutations for the above two configurations due to the symmetry of the coupling architecture. Figures 5(c) and 5(d) depict the AD to OD transition for these two OD configurations as a function of $K$. The transition occurs at $K=17.33$ for both cases, i.e., the IHSS is created and stabilized. Increasing the coupling strength further, the stable IHSS becomes unstable at $K=17.36$ and 40.41 for the $\alpha \alpha \beta$ and $\alpha \beta \gamma$ patterns, respectively. For more than $N=3$ identical oscillators, the AD to OD transition is generically produced, but the OD configurations after the transition are much more complex.

In conclusion, the $\mathrm{AD}$ to $\mathrm{OD}$ transition phenomenon has been systematically identified in nonlinear oscillators with time-delayed, dynamic, and conjugate couplings, which are so far understood as having the tendency to induce AD alone. We have shown that both AD and OD can be stabilized by a time delay in the same system of coupled oscillators that does not experience either AD or OD in the absence of time delay. However, for this coupling scenario, the AD to OD transition cannot occur if the coupled oscillators are identical. Instead, time-delayed coupling can facilitate its occurrence for a low degree of heterogeneity present in the system. Interestingly, we have also shown that the AD to OD transition can be clearly established in systems of identical oscillators with dynamic and conjugate couplings. Furthermore, we have found the occurrence of a stable HSS (AD) coexisting with a stable IHSS (OD) for conjugate coupling after the transition. In the general case for $N>2$ identical oscillators, the transition between the AD and OD regimes is sustained, however, a rich manifestation of stable OD configurations has been revealed. A detailed characterization of these IHSS solutions awaits further investigations. Even though here we have studied the $\mathrm{AD}$ to $\mathrm{OD}$ transition for the paradigmatic model of coupled Stuart-Landau oscillators, we firmly expect that the transition scenario described here will essentially characterize the general features of such a transition in various physical and biological systems. Our study of the AD to OD transition should gain significant implications in diverse applications not only in real-world systems, but also for control studies and engineering problems [28,29].

This work was supported by the National Natural Science Foundation of China under Grant No. 11202082, the Fundamental Research Funds for the Central Universities of China under Grant No. 2013QN165, the SUMO (EU), and IRTG1740 (DFG-FAPESP).
[1] Y. Kuramoto, Chemical Oscillations, Waves, and Turbulence (Springer, Berlin, 1984).

[2] M. Lakshmanan and D. V. Senthilkumar, Dynamics of Nonlinear Time-Delay Systems (Springer, Berlin, 2010).

[3] G. Saxena, A. Prasad, and R. Ramaswamy, Phys. Rep. 521, 205 (2012).
[4] A. Koseska, E. Volkov, and J. Kurths, Phys. Rep. 531, 173 (2013).

[5] A. Koseska, E. Volkov, and J. Kurths, Phys. Rev. Lett. 111, 024103 (2013).

[6] D. G. Aronson, G. B. Ermentrout, and N. Kopell, Physica D 41, 403 (1990). 
[7] D. V. Ramana Reddy, A. Sen, and G. L. Johnston, Phys. Rev. Lett. 80, 5109 (1998).

[8] W. Zou and M. Zhan, Phys. Rev. E 80, 065204(R) (2009).

[9] W. Zou, D. V. Senthilkumar, M. Zhan, and J. Kurths, Phys. Rev. Lett. 111, 014101 (2013).

[10] K. Konishi, Phys. Rev. E 68, 067202 (2003).

[11] R. Karnatak, R. Ramaswamy, and A. Prasad, Phys. Rev. E 76, 035201(R) (2007).

[12] I. Prigogine and R. Lefever, J. Chem. Phys. 48, 1695 (1968).

[13] K. Bar-Eli, Physica D 14, 242 (1985).

[14] A. Kuznetsov, M. Kaern, and N. Kopell, SIAM J. Appl. Math. 65, 392 (2004).

[15] A. Koseska, E. Volkov, A. Zaikin, and J. Kurths, Phys. Rev. E 75, 031916 (2007).

[16] M. Y. Kim, R. Roy, J. L. Aron, T. W. Carr, and I. B. Schwartz, Phys. Rev. Lett. 94, 088101 (2005).

[17] Y. Zhai, I. Z. Kiss, and J. L. Hudson, Phys. Rev. E 69, 026208 (2004).

[18] A. Koseska, E. Ullner, E. I. Volkov, J. Kurths, and J. GarcíaOjalvo, J. Theor. Biol. 263, 189 (2010).
[19] N. Suzuki, C. Furusawa, and K. Kaneko, PLoS ONE 6, e27232 (2011).

[20] Y. Goto and K. Kaneko, Phys. Rev. E 88, 032718 (2013).

[21] R. Curtu, Physica D 239, 504 (2010).

[22] K. P. Zeyer, M. Mangold, and E. D. Gilles, J. Phys. Chem. 105, 7216 (2001).

[23] C. R. Hens, O. I. Olusola, P. Pal, and S. K. Dana, Phys. Rev. E 88, 034902 (2013)

[24] H. Daido and K. Nakanishi, Phys. Rev. Lett. 96, 054101 (2006).

[25] D. M. Abrams and S. H. Strogatz, Phys. Rev. Lett. 93, 174102 (2004).

[26] D. Breda, Appl. Numer. Math. 56, 305 (2006).

[27] D. Breda, S. Maset, and R. Vermiglio, Appl. Numer. Math. 56, 318 (2006).

[28] Y. Tang, H. Gao, W. Zou, and J. Kurths, IEEE Trans. Cybern. 43, 358 (2013).

[29] Y. Tang and W. K. Wong, IEEE Trans. Neural Networks Learn. Syst. 24, 435 (2013). 